\title{
INVESTIGACIONES
}

\section{HOMOGENEIDAD Y HETEROGENEIDAD: UN ESTUDIO COMPARATIVO SOBRE LA REFORMA EDUCATIVA DE LA DÉCADA DEL 90 EN ARGENTINA, BRASIL, CHILE Y MÉXICO*}

\author{
Homogeneity and Heterogeneity: A comparative study of educational reform \\ in the decade of the 1990s in Argentina, Brazil, Chile and Mexico
}

\section{Nora Rut Krawczyk $k^{1}$, Vera Lúcia Vieira ${ }^{2}$}

\author{
${ }^{1}$ Facultad de Educación, Universidad Estadual de Campinas - UNICAMP (San Pablo, Brasil). \\ Dirección postal: Rua Navarro de Andrade 140, apto. 92. CEP: 05418-020 - San Pablo/SP. Brasil. \\ E-mail: norak@unicamp.br \\ ${ }^{2}$ Departamento de Historia de la Facultad de Ciencias Sociales de la Pontificia Universidad Católica de San \\ Paulo - PUC/SP (Brasil). Dirección postal: Dr. Veiga Filho 83, apto. 142. Higienopolis - San Pablo/SP. Brasil
}

\section{Resumen}

Un estudio comparado sobre los impactos de la reforma educativa de la década del 90, en Argentina, Brasil, Chile y México, a partir del análisis de la producción académica. Fueron analizadas 186 producciones así distribuidas: Argentina (45), Brasil (62), Chile (36) y México (43).

El estudio enfoca la reconfiguración de la gestión de la educación a partir de la nueva lógica de regulación social y del nuevo papel del Estado y revela la relación entre los cambios sociales y educativos y la historicidad de cada uno de esos países.

Se destacan los aspectos que dejan en evidencia la homogeneidad de la reforma educativa en la región y las especificidades nacionales que interfieren en su concretización.

Se pone en evidencia que la historicidad que caracteriza la realidad educativa en estos países viene sufriendo, en México una "ruptura conservadora"; en Chile, una "continuidad conservadora”; en Brasil, una renovación conservadora, y en Argentina, una ruptura interrumpida.

Palabras clave: América Latina, regulación, Reforma educativa, gestión de la educación

\begin{abstract}
A compartive study of the impacts of educational reforms in the decade of the 1990s, in Argentina, Brazil, Chile and Mexico, base on the análisis od academic production. One hundred and eighty-six (186) publications were analyzed distributed in the following manner: Argentina 45, Brazil 62, Chile 36, and Mexico 43.

The study focused the reconfiguration of the administration of education from the perspectiva of the new logia of social regulation and the new role of the State and reveals the relationship between social and educational changes and the historicity of each one of those countries.

Apparent are those aspects that evidence the homogeneity of educational reforms in the region and the national specificities that infere in its realization.

It is evident that the historicity that characterizes the educational reality in those countries underwent in Mexico a "conservative break", in Chile a "conservative continuation", in Brazil "a conservative renovation", and in Argentina "an interrupted break".
\end{abstract}

Key words: Latin America, regulation, educational reform, administration of education.

* La investigación que dio origen a este artículo fue realizada con el apoyo de la FAPES/Brasil y CNPq/Brasil. 


\section{INTRODUCCION}

En la década del 90 se iniciaron en América Latina reformas educativas junto con transformaciones profundas en las esferas de la economía, de las instituciones sociales, culturales y políticas, así como en la naturaleza de las relaciones entre estas diferentes esferas. Tuvieron como finalidad no sólo la expansión de la enseñanza, sino también la adecuación de la educación pública a los cambios de la lógica de regulación capitalista.

En casi todos los países estas reformas resultaron, en gran medida, de un proceso de inducción externa, articulado con las políticas de organismos internacionales de préstamos para la región. La necesidad de esa reforma fue justificada mediante la publicación de investigaciones que evidenciaron los logros y las deficiencias de los sistemas educativos teniendo en cuenta las condiciones de la reestructuración del sector productivo y de los cambios institucionales que alteraron la estructura del Estado y de las relaciones sociales, en el ámbito de un nuevo orden mundial.

En esos estudios se indicó que el crecimiento de la matrícula escolar en la región, durante los años 60, fue concomitantemente a la disminución de la calidad del sistema, más aguda aún en los años 80. La ausencia de una gestión del presupuesto, de una organización institucional adecuada y de una propuesta pedagógica de acuerdo con la heterogeneidad sociocultural de la población incorporada habría generado la ineficiencia del sistema en responder a las necesidades propias del capitalismo en este momento histórico.

El diagnóstico y los cambios propuestos para la educación en América Latina en este período fueron elaborados a partir de las necesidades de reformulación del carácter regulador del Estado y de la fuerte crítica a las funciones de los estados nacionales de modelo keynesiano, en el marco de una nueva etapa del capitalismo.

Pasados diez años de la implementación de la reforma, las investigaciones efectuadas por los especialistas en los diferentes países muestran el fracaso de las promesas que sus ideólogos hicieron a la sociedad. También queda claro, en estos estudios, que la uniformidad de la política educativa, en escala global, está vinculada al creciente peso de las agencias internacionales y del liderazgo del Banco Mundial en el diseño y en la ejecución de la reforma educativa en los países en desarrollo.

El conjunto de las investigaciones deja evidencias de que estas reformas tienen un carácter homogéneo y homogeneizador tanto en la comprensión de las realidades nacionales como en sus propuestas, estandarizando la política educativa en la región.

Al reflexionar sobre los aspectos particulares de las condiciones de desarrollo de los países, entretanto, se observa que esta reforma se procesó en sociedades con historias diferentes que, de alguna forma, intervienen en la concretización de los cambios, lo que le conferiría específicas situaciones nacionales. Esto no invalida que la reforma educativa haya producido rupturas institucionales que fragilizan prácticas sociales históricamente constituidas.

El estudio de las realidades nacionales a partir de una perspectiva analítica y explicativa posibilita comprender la historicidad de las categorías de análisis en los diferentes países y desentrañar la interpenetración que se da entre los niveles locales y globales, reestructurando las culturas institucionales. Este propósito nos llevó al análisis de las investigaciones sobre la reforma educativa en la década del 90 en Argentina, Chile, 
Brasil y México y a realizar un estado del arte para comprender la especificidad de la producción del conocimiento en cada uno de estos países y en la concretización de la reforma (Krawczyk, Vieira 2005) ${ }^{1}$.

En este trabajo analizaremos, en los procesos de transformación ocurridos en estos países, los cambios en la organicidad del sistema educativo que homogenizan las situaciones nacionales, así como sus particularidades. Entendemos como organicidad la dinámica por la cual se procesan las relaciones de poder en la organización y gestión del sistema educativo y de la escuela. Presupone este concepto la historicidad de las formas como fueron concretizándose, en el espacio público, los intereses de los diferentes grupos sociales.

\section{HOMOGENEIDAD Y HETEROGENEIDAD EN LA REFORMA EDUCATIVA}

La implementación de las reformas educativas en América Latina desencadenó un conocimiento crítico relevante que se dedica a la comprensión de la situación actual de la educación en la región. Estos estudios muestran que se configuró, en los años 90, a través de la política de descentralización, la relación entre el Estado y la sociedad, substituyendo su papel de proveedor directo de bienes y servicios y de control centralizado del conjunto de las actividades sociales por funciones de coordinación y regulación legal, dentro de las cuales la educación adquiere relevancia.

Al reunir los datos de estas investigaciones es posible observar que en su mayoría destacan que la descentralización tuvo en común, en los diferentes países, un objetivo financiero vinculado a la crisis fiscal de los gobiernos nacionales, que se expresa en un nuevo escenario de distribución de responsabilidades y de dinámicas de negociación. Las investigaciones dejan en evidencia la descentralización de las funciones administrativas para las instancias locales y para las instituciones escolares, centrada en la transferencia de competencias, paralelamente a un proceso de centralización del poder de decisión y del control en los gobiernos nacionales. Esta centralización es identificada en las investigaciones por la concentración de las instancias legales y de evaluación del sistema en los gobiernos centrales (González; Arango 1997; Kisilevsky, S/D; Rivas 2004; Riquelme 2004; Calvo Pontón 1997; Pardo 1999; Donoso 2004; García-Huidobro 1999, 2004; García-Huidobro, Cox, 1999; Gluz 2001; Davies 1999; Bassi 1996; Oliveira 2004; Guimarães 2004).

En el ámbito escolar, esta lógica de la regulación consolidó una nueva relación entre el Estado y la sociedad que consubstanció el cambio del tenor de la participación por la corresponsabilización de los individuos en el abastecimiento y en la calidad de la educación. El compartir los deberes fue incentivado por los gobiernos nacionales

\footnotetext{
"El estado del arte de las investigaciones sobre la Reforma Educativa en la década del 90, en Argentina, Brasil, Chile y México", concluido en 2005, fue coordinado por Nora Rut Krawczyk y con la participación como investigadora principal Vera Lúcia Vieira. El estudio se concentra en el análisis de la producción académica sobre la organización y gestión del sistema educativo y de la institución escolar, dimensiones privilegiadas en la reforma. Para la realización del estado del arte fueron analizadas 186 producciones, distribuidas entre los países de la siguiente manera: Argentina (45); Brasil (62); Chile (36); México (43).
} 
con la definición de mecanismos que tenían como objetivo la participación de los diferentes segmentos sociales en el plano local y, principalmente, en el gerenciamiento de las escuelas. Esto ocurre porque, para dar posibilitad de repasar los recursos para las escuelas de forma diferenciada y vinculada a las demandas de la comunidad escolar, los gobiernos solicitan una propuesta pedagógica elaborada colectivamente que, además de las necesidades, expliciten también las metas y estrategias de acción. En el análisis de la concretización de la elaboración del proyecto escolar se observa que la política oficial valoriza la particular capacidad de administrar los problemas y captar los recursos necesarios, además de movilizar la comunidad para ayudar a las escuelas a resolverlos, como expresión de la autonomía escolar.

Según las investigaciones, la incorporación del modelo gerencial y la adopción de nuevas estrategias de inversiones en la educación revelan la implementación de la lógica de mercado, perceptible también en los valores y prácticas identificados en las instituciones (Dussel, Tiramonti, Birgin 1998; González 1999; Figueroa 1997; Viriato 2001).

El discurso participativo, enfatizado en el modelo gerencial produjo dos fenómenos que afectaron la dinámica escolar. Por un lado, ocurrió la burocratización del cotidiano escolar y la participación de las instancias colegiadas, aumentando la actividad administrativa, las demandas de las familias y provocando un cambio del perfil del trabajo escolar. Al mismo tiempo que se fortaleció la figura del director en cuanto a ser administrador, distanciándolo de las actividades pedagógicas, se observa el deterioro de la producción colectiva de los docentes, agravada por la instabilidad en el empleo.

Por otro lado, el aumento de las responsabilidades de las instituciones escolares y de las familias en la gestión financiera de la institución y en las estrategias de resolución de los problemas educativos produjo un activismo institucional y una relación de competitividad entre las escuelas, dada la constante necesidad de elaboración de proyectos, de contactos y convenios con la comunidad para concurrir a nuevos recursos gubernamentales y privados (Birgin 2000; Carnoy 1998; Oliveira, Duarte 1999; Teixeira 1998; Fonseca 2003).

Al analizar este proceso las investigaciones indican que el espacio de autonomía construido a partir de estas políticas queda restringido a las responsabilidades transferidas a las instituciones escolares como consecuencia del proceso de descentralización, pero no se configuran como transferencia efectiva de poder.

Observan, además, que la nueva dinámica establecida coloca a las instituciones con menos recursos -materiales, humanos e institucionales- en peores condiciones para elaborar proyectos de calidad, produciendo la atomización de las instituciones y fortaleciendo la reproducción de las desigualdades sociales (Tiramonti 2004; Arroyo 2004, Poliak 2004; Mizala Romaguera, Fareen 1998; Villegas 1999; Fonseca 2003).

Ese proceso común de descentralización de la educación se concretó de manera diferente debido a la historicidad de cada país de la región con relación, principalmente, a la lógica de configuración de los sistemas educativos nacionales y de las coyunturas nacionales en las cuales se engendra la reforma educativa regional que estamos analizando. Son circunstancias que expresan tanto los procesos de ruptura y continuidad emergentes del período anterior tanto cuanto los nuevos embates sociales, políticos y económicos nacionales e internacionales.

En Argentina, el nuevo papel del Estado que daría soporte a la descentralización fue traspasado, a lo largo del período, por la fuerte crisis económica que se instaló en el país en la década del 90. 
Según las investigaciones, en la reforma educativa argentina se abandonó el carácter universalista del papel del Estado en el abastecimiento de la educación pública, ganando importancia el carácter compensatorio de las políticas nacionales (Dustchatzky, Redondo 2002).

Desde la constitución del sistema educativo, en el siglo XIX, la democratización de la educación formal fue asociada en este país al carácter universalista del Estado, apuntando a la universalización de la enseñanza primaria para atender a los intereses nacionales de homogeneización cultural y a los intereses de los sectores emergentes (constituidos, principalmente, por inmigrantes) en relación a la promoción y a la participación política (Weinberg 1987; Tedesco 1982; Puiggrós 1990).

Este cambio en la función social del Estado nacional aparece con mucha fuerza en las investigaciones que analizan el Plan Social de la Educación (PSE), por haber sido esta la política más importante del gobierno, destacándose las consecuencias de su naturaleza asistencial en el cambio de los comportamientos institucionales.

El PSE fue la única política nacional dirigida directamente a las escuelas de todo el país, que tuvieron los mayores índices de población pobre en la década del 90². Las investigaciones de los estudiosos clarificaron cómo el Plan fue inicialmente gestado para la atención de un tipo de población, cuyas condiciones de pobreza estaban vinculadas a las características estructurales de la sociedad, pero este universo fue siendo significativamente ampliado por la necesidad de la inclusión de un contingente cada vez mayor de la población afectada por la crisis económica. El empobrecimiento general de la población también afectó las políticas de distribución de las competencias educacionales, que aumentaron las responsabilidades de las instituciones escolares y de las familias. Las investigaciones constataron, en los últimos años de la década del 90, el crecimiento de la demanda familiar sobre la escuela y el Estado para la resolución de los problemas, siendo estos no sólo vinculados a las cuestiones materiales, sino también a los comportamientos sociales, tales como la violencia (Kessler 2002; Tiramonti 2004).

Concluyen que las prácticas que consolidan el PSE en las escuelas acaban produciendo comportamientos discriminatorios de la pobreza, en el lugar de la función integradora que sustentaba la lógica universalista anteriormente vigente (Dussel, Tiramonti, Birgin 1998; Tiramonti 2001).

Hay consenso respecto de que el fortalecimiento de la compensación y del presupuesto público selectivo en el comportamiento regulador del gobierno nacional en la educación pública era parte de un conjunto de estrategias vinculadas, de un lado, a la disminución de los gastos públicos y, de otro, a la necesidad de generar estrategias de contención social, dado que la población, afectada por el ajuste estructural, venía manifestando su descontento y exigiendo al gobierno nacional urgencia en la resolución de problemas derivados de su rápida pauperización (Barreyro 2001).

Una de las preocupaciones que orientan al análisis de las investigaciones en la Argentina sobre los cambios en el papel del Estado es el abandono de su carácter universalista.

2 Las inversiones para la implantación del PSE, a pesar de ser realizadas con recursos propios del gobierno nacional, cumplieron la función de contrapartida a los préstamos de los organismos internacionales para otros programas. 
En el caso de Brasil ocupa su lugar la ausencia de descentralización del poder en la nueva configuración que el Estado asumió. Según las investigaciones, se creó en este país una lógica de centralización del poder en el gobierno nacional, a través de un conjunto de normas, como el sistema de evaluación nacional en el Fondo de Desarrollo de Educación Fundamental ${ }^{3}$ y Valorización del Magisterio, FUNDEF, que pasaron a regular la implantación de la municipalización.

La descentralización propuesta por el gobierno nacional adquirió un significado específico en el caso de este país, porque acabó normatizando nacionalmente una diversidad de redes que configuraban la educación pública desde su inicio, en el siglo XX, cuando los estados asumieron la responsabilidad de la educación primaria. Tal diversidad se mantuvo en el período centralizador de la dictadura de la segunda mitad del siglo XX y se acentuó durante la democratización, después de la década del 80, con la definición de las relaciones entre las tres instancias federativas (unión, estados y municipios) que delegó a estos dos últimos la responsabilidad de asumir la Educación Infantil, Fundamental y Media.

La política educativa más discutida por los investigadores fue la municipalización de la Educación Fundamental. Esto debido a las alteraciones que provocó la distribución de competencias y atribuciones entre las diferentes esferas del poder público en las diferentes redes de educación pública y las expectativas de autonomía que fueron construyéndose socialmente en contraposición al período dictatorial (Bezerra 1997; Costa 1997).

En México se configuró un Nuevo Federalismo que rompió con la idea del Estado nacional como único responsable de la educación pública, diferente de lo que ocurría en los otros países analizados. Se creó un sistema educativo nacional centralizado ya en el siglo XIX, que apuntaba hacia una homogeneidad educacional y cultural de la población para la consolidación de la Nación y de una sociedad democrática liderada, en el esfuerzo posrevolución, del Partido Revolucionario Institucional (PRI), llegando de allí su fuerte connotación laica y republicana.

La constitución de ese Nuevo Federalismo fue expresión de una crisis de hegemonía en todos los ámbitos de la Nación, que resultó, en un consenso nacional sobre la necesidad de la descentralización, lo que permitiría la recomposición de los factores sociales en la disputa por el poder. Así como en Brasil, aunque por razones diferentes, la preocupación de los investigadores está en identificar la descentralización del poder.

Los análisis constatan que, coherentemente con la tendencia regional, se mantuvo la fuerte centralización del gobierno nacional, a través de funciones normativas y de evaluación. Al mismo tiempo, se evidencia el mantenimiento de la hegemonía del SNTE en el interior del poder central y su liderazgo en la gestión educativa del país (Street 2001; Calvo Pontón 2002).

Un espacio aún privilegiado por el gobierno nacional en la época de la investigación era la responsabilidad por la implementación de programas compensatorios orientados al grupo de extrema pobreza, manteniendo la tradición de garantizar que las escuelas llegasen a cualquier lugar del país. La producción académica centralizó su atención en

3 La Educación Fundamental tiene 8 años de duración y la edad obligatoria es de 7 a 14 años. 
la evaluación de los resultados de estos programas en su nueva dimensión social, o sea, la de mejora de la calidad de la red.

Los diferentes diagnósticos indican que no está disminuyendo la desigualdad educativa, aunque reconozcan aspectos positivos en lo que respecta a las finalidades de los programas. A pesar que no se hayan notado cambios en el rendimiento ni en la adecuación de los programas y prácticas pedagógicas de la organización multiseriada de las escuelas, reconocen que los incentivos a los docentes han estimulado su fijación en las regiones rurales de extrema pobreza y el acceso de los alumnos al material didáctico.

Es bastante presente en la investigación en México la preocupación del comportamiento institucional de las escuelas frente a la desigualdad social, teniendo como referencia el principio de equidad educativa. El resultado de las investigaciones apunta que la casi universalización aún presenta problemas serios de repetición y deserción y que la calidad de la enseñanza está distribuida de forma desigual entre las regiones y clases sociales (Quiroz 2000; Bracho 1997). Una de las cuestiones destacadas por algunos investigadores es que la segmentación en el interior de la escuela pública no es resultado de la implementación de estrategias homogéneas en situaciones heterogéneas. La situación es mucho más grave; lo que sucede es que el sistema educativo, en su funcionamiento cotidiano, está proporcionando, tanto en términos cuantitativos cuanto cualitativos, insumos diferentes a los distintos contextos sociales, reproduciendo y consolidando la diferenciación (Schmelkes, 1995).

Ya en Chile, que siguió un camino diferente en relación a los otros países en la década del 90, no se asistió ni a una reestructuración del Estado (como en México) ni a una disputa por la hegemonía de diferentes proyectos de Estado (ejemplo de Brasil y Argentina) que afectase la política educativa. Por el contrario, ya había allá un Estado mínimo sustentado en la lógica de regulación de mercado, aún no incorporado por el sector educativo (Nunes 1994, 1999, 2004). Los investigadores demostraron que en la década del 90 el Estado nacional reconfiguró el sistema de subvenciones y desarticuló al sector (Vargas 1997), semejante a la lógica ya vigente en las otras áreas sociales que apuntaban a ese objetivo, reservándose el gobierno nacional la resolución de la falta de equidad vigente en la educación.

La desregulación del sector educativo afectó el trabajo de la escuela, y particularmente la condición de los docentes, de forma más contundente de lo que ocurrió en los otros países, debido a las estrategias adoptadas por el gobierno chileno (Pereira 2000; Serrano \& Fernández 2001; Díaz 2002; Espinoza 1997).

En Chile, el trabajo docente sufrió un proceso de desregulación equivalente al vivido por las otras categorías de asalariados en décadas anteriores. Las investigaciones constatan la flexibilización contractual y salarial (Carnoy 1998, Díaz 2002), el vaciamiento de las negociaciones colectivas, la pérdida de la estabilidad y la desvinculación de las contrataciones con el estatuto del magisterio (Miranda, De Simone, 2002).

Aunque la desregulación del trabajo haya afectado al conjunto de los profesionales de la educación, en las escuelas con más subvención no se configuró el deterioro del trabajo escolar (Mizala, Romaguera 2000; Petruzzi 2000). Al mismo tiempo, esta lógica de subvención, junto con la desregulación del trabajo, estimula la competición entre los profesionales de la educación de forma más contundente que la observada en los otros países.

No hay acuerdo entre los investigadores ni en la sociedad sobre las consecuencias de esta situación mencionada, una vez que los análisis son marcados por el antagonismo 
ideológico (Illanes, Lagos 1998, Monzón 2000; Escoffier 1995; Baeza 1997; Mizala 1997; Romaguera 1998). Además de esto, las investigaciones muestran que la idea de que la equidad en el sistema educativo podría ser garantizada por la inversión pública que regulase las diferenciaciones sociales e institucionales no se concretizó (Figueroa 1997; Sanhueza 1999; Mizala, Romaguera 1998), aunque el gobierno chileno haya realizado una fuerte inversión en políticas de discriminación positiva, extendidas gradualmente a todo el sistema educativo (Cardona 2000; Prado, Gajardo 1995; San Miguel 1999).

El proceso de descentralización en cada uno de los países, al configurar un nuevo papel del Estado nacional, alteró las relaciones de poder entre el gobierno central y los locales, lo que, a su vez, la constituye en una dimensión fundamental en la institución de la nueva organicidad de los sistemas educativos.

En el caso de Argentina, las alteraciones en la estructura del sistema y la finalización de la transferencia del servicio educativo iniciado por los gobiernos dictatoriales, a lo que se sumó la preexistencia de un federalismo centralizado, forman la nueva organicidad del sistema educativo.

En el inicio de la década del 90 la transferencia de las escuelas de Educación Infantil, Secundaria y Superior, hasta entonces sobre la responsabilidad del Ministerio de Cultura y Educación (MCyE), consolidó la descentralización de la responsabilidad de proveer educación formal y de la casi totalidad del financiamiento del sistema educativo para las Provincias ${ }^{4}$. La división de responsabilidades y compromisos de financiamiento para la implementación de la reforma educativa entre la nación y las provincias fue objeto del Pacto Federal Educativo (PFE). Entretanto, la política educativa se transformó, durante todo el período, en un espacio de negociaciones y de tensiones con las provincias.

Se observa, además, la transferencia de la lógica a las gestiones provinciales, que en algunos casos convive con el mantenimiento de una fuerte dependencia en relación al gobierno nacional. Esta vinculación deriva no sólo de la centralización tributaria, sino también, en un primer momento, de la centralización del poder en la definición de prioridades de inversiones de gran monto de recursos originarios de los organismos internacionales para la reforma educativa (Rivas 2004).

Añaden los estudios que, mientras el gobierno nacional disponía de estos recursos, lo que le permitiría, entre otras cosas, componer un cuerpo técnico competente para definir la reforma, fue mínima la posibilidad de debate y negociación de la mayoría de las provincias. Esta situación se deterioró frente a la fuerte crisis financiera que asoló al país, con la fragilidad del poder central, que se vio imposibilitado de continuar implantando políticas, y la dificultad de los gobiernos provinciales de sustentar propuestas alternativas (Vior 1999; Tiramonti 2001).

El carácter centralista y camuflado que caracterizó todo este proceso de negociaciones con las provincias se manifiesta también en las gestiones educativas provinciales (Tedesco, Tenti Fanfani 2001). En muchas de ellas, esta gestión centralizada implicó el desmantelamiento de las instancias colegiadas, diferentemente de los otros países, en los cuales fue propuesta una organización administrativa que contemplase un espacio de decisiones colectivas (Vior 1999; Rivas 2004).

4 Durante el gobierno militar (de 1976 a 1982) fueron transferidas a las provincias todas las escuelas de Educación Primaria y la mayor parte de las de Secundario. 
Los cambios en la estructura del sistema educativo significan la substitución de la organización tradicional (primaria, de siete años, y secundaria, de cinco) por un ciclo de Educación Inicial de dos años (cuatro y cinco años de edad), otro de Educación General Básica (EGB), de nueve años, y el nivel polimodal, de tres años, extendiéndose la obligatoriedad de siete para diez años. Este cambio, que se suponía radical y de compleja implementación, perdió su sentido consolidándose la heterogeneidad del servicio educativo en el país (Tedesco, Tenti Fanfani 2001).

Algunos investigadores consideran que la nueva estructura del sistema que se pretendió implementar no era esencial para resolver los problemas educacionales y, al mismo tiempo, demandaba un montante de recursos y de acciones inexistentes en aquel momento.

Las investigaciones sustentan que, cuando implantada la EGB3 a continuación del antiguo primario, hubo un aumento de la permanencia de los adolescentes en la escuela (Duschastzky 1999; Krichesky, Cappellacci 1999). Sin embargo, la implementación de la nueva estructura no fue acompañada por la correspondiente formación docente ni por la adecuación de los espacios institucionales y condiciones administrativas necesarias para hacer frente a las nuevas demandas (Romagnoli, Romagnoli 2002; Oyola 1998).

La heterogeneidad de situaciones provinciales que se consolidaron en el marco de la crisis de hegemonía del Estado nacional resulta, según las investigaciones, en una organicidad que tiene como principal característica la fragmentación y la reproducción de la desigualdad (González, Arango 1997; Tiramonti 2001; Rivas 2004). En los análisis sobre la gestión escolar, las investigaciones muestran que la incorporación de una lógica de mercado de transferencias de responsabilidades (antes afectas al Estado) para las escuelas y las familias profundiza esa fragmentación de la diversidad de culturas institucionales y de las características socioeconómicas y culturales de la comunidad.

En México la organicidad del sistema educativo, inherente al nuevo federalismo, conforme ya dijimos, evidenció la reciente dinámica que alteró la relación entre el gobierno central y los regionales y legitimó diferentes factores políticos, al mismo tiempo que se recompusieron las fuerzas tradicionales. En este contexto, la política de descentralización a ser desencadenada por la reforma educativa, que se pretendía innovadora, quedó subordinada a la lógica de la política partidaria, en la cual el corporativismo de SNTE se mantuvo predominante (Loera, Sandoval 1999; Calvo Pontón 2002; Loyo, Muñoz 2001; Ornelas 1999).

Esta dinámica de negociación, entre el gobierno central y los gobiernos provinciales, fue intensa por la falta de una política fiscal que posibilitase dar visibilidad a los criterios utilizados para las transferencias de recursos económicos a la educación.

El control centralizado de estos recursos llevó, según los especialistas, a una fragmentación de las negociaciones por el financiamiento educativo, lo que resultó en el fortalecimiento del gobierno central y de aquellos estados con más posibilidades de negociación, técnica o política. Resultando de esto, según las investigaciones, que las transferencias federales de los recursos a los estados no hayan apuntado, de hecho, a la reducción de las desigualdades regionales (Esquivel 1999; Latapí, Herrero 2000).

Tanto en México como en Argentina es posible observar que la historicidad de sus dinámicas federalistas se manifestó en la dificultad de institucionalizar una nueva normatización redistributiva, sometida a una negociación política constante.

En México, además del análisis de la dinámica distributiva de la descentralización y sus consecuencias, un vasto conjunto de investigaciones enfoca la importancia de la 
descentralización en cuanto al eje principal de la viabilidad de innovaciones, acompañando los presupuestos de la reforma en América Latina. Según varios autores, en ese país la descentralización abrió espacio para que germinasen condiciones políticas, técnicas y financieras de innovación en distintos planos de la organización y estructura de las redes educativas locales (Pardo 1999; Camacho s/d).

Los estudios sobre las políticas educativas locales, permiten constatar la diversidad de situaciones existentes en el país, tanto en lo que se refiere al carácter ideológico de las propuestas cuanto a la diferenciación de las posibilidades concretas de su implantación, como se verificó en otros países (Espinoza 1999; Carmona 1996).

Uno de los temas comunes encontrados en las investigaciones sobre las realidades locales es el papel de la supervisión en las dinámicas de los sistemas provinciales y de las instituciones escolares.

La supervisión se colocó con fuerza en la década del 90, como uno de los tópicos destacados en los debates sobre la gestión de la educación. Esta preocupación demostraba el interés en hacer efectivos cambios en el perfil de esta categoría, de manera que promoviese la implantación de la reforma en las escuelas. Reconociendo, así, que la supervisión ocupaba una posición estratégica en la estructura del sistema educativo mexicano, tanto en la articulación entre la administración central, sea nacional o estatal, y las unidades escolares como en las dinámicas institucionales de las escuelas. La integración del SNTE con la Secretaría de Educación Pública y con las instituciones escolares, principalmente por medio de la supervisión escolar, produjo una lógica de control bastante compleja del sindicato sobre la vida cotidiana en las escuelas y consecuentemente en los docentes.

En las investigaciones se observa que la supervisión, a pesar de su fuerza institucional, dirige sus acciones a las actividades medio, perdiendo la perspectiva de las finalidades de su función propiamente pedagógica. El burocratismo en el que se encuentran envueltos es útil para el mantenimiento del control de la escuela por el sindicato y para la mediación entre ésta y la administración central (Espinoza 1999; Carmona 1996).

Indican, aún, que el discurso de la necesidad de reposicionar la función pedagógica del supervisor, elogiado por las secretarías de educación nacional y estatales, no se materializó en la relación que ellas establecieron con la supervisión, lo que reforzó el carácter burocrático de la función del supervisor.

Las investigaciones que abordan la dinámica escolar, certifican el aislamiento y el individualismo de la actividad docente, dadas las condiciones de trabajo y la dinámica burocratizada de la escuela (Sandoval 2002; Ezpeleta 2003). Una de las políticas que vienen afectando más esta dinámica escolar es, según los investigadores, el Programa de Escuela de Calidad (PEC). Según la bibliografía, el PEC refleja algunos de los trazos más importantes del acuerdo empresarios-gobierno, partiendo de la suposición que las escuelas mejoran más rápido cuando se ofrece a su dirección y al cuerpo docente la posibilidad de recibir recursos extras para implantar un proyecto escolar propio. Al orientar su finalidad para la competición por la obtención de financiamiento para la escuela se alteró la dinámica implantada anteriormente por el Programa de Gestión de la Escuela Primaria (PGEP), que priorizaba la reflexión de la gestión pedagógica para mejorías de la calidad de la enseñanza (Bonilla 2002; Aboites, s/d; Ezpeleta 2003).

Las investigaciones constatan el delineamiento de una nueva dinámica institucional a partir del PEC, común a los otros países de la región, de incremento del burocra- 
tismo en detrimento del aspecto pedagógico. También registran que el desempeño de las instituciones depende de la capacidad del equipo docente de elaborar proyectos y captar recursos, reproduciéndose, así, la desigualdad en el interior del sistema educativo (Bonilla 2002; Martínez 2003).

La nueva lógica para la gestión escolar implantada por el PEC vinculó la posibilidad de participación de las escuelas en el Programa a la constitución de consejos escolares y a la participación de los diferentes segmentos en la elaboración de los proyectos escolares. Las investigaciones dejan en evidencia una disociación entre el discurso de las autoridades escolares y la práctica de participación de los padres, además de una heterogeneidad en las formas de funcionamiento de los consejos y de compromiso de estos en la escuela (Loera 2003; Martínez 2003).

Las prácticas de participación de la comunidad en la institución escolar tienen origen en la revolución mexicana, con la movilización ocurrida a favor de la universalización de la enseñanza primaria. Estas prácticas tendieron, principalmente, para el compromiso de los padres en el mantenimiento de las escuelas y en la implementación de los programas compensatorios (Rockwell 1997). Las investigaciones demostraron que muchas de estas prácticas permanecen, pero con un nuevo sentido. La percepción que los padres de menor poder adquisitivo son los que más contribuyen, como consecuencia de la omisión del Estado en las escuelas más carentes, lleva a los autores a reflexionar sobre la falta de equidad y la no gratuidad de la escuela pública (Mercado 1997). Se destaca también que la participación de los padres en las políticas compensatorias viene a "compensar" la ausencia del Estado en la provisión de las condiciones necesarias para el funcionamiento de las escuelas y en el control de la implantación de los programas (Ezpeleta, Weiss 2000).

Podemos extraer, del análisis de los autores, lógicas diferentes en el estudio de la relación entre la comunidad y la escuela. Una manifiesta las consecuencias de la ausencia del carácter movilizador de la participación de las familias, propia de la tradición constituida en el período revolucionario y sus consecuencias para la gratuidad e equidad de la enseñanza. Inicialmente, la comunidad integraba de forma espontánea el esfuerzo de la Nación en proveer educación, independientemente de la institucionalización o legalización de esta relación, pero, progresivamente, las escuelas más pobres pasaron a depender de la contribución de las familias de los alumnos.

La otra lógica que se expresa en las investigaciones es el estudio de la relación entre la participación y su institucionalización, identificando que ésta materializa la propuesta de "democratización del poder" vía burocracia participativa.

Por fin la tercera analiza la configuración de la participación social y constata que, inicialmente orientada para la democratización de las relaciones de la escuela, se propone la liberalización de las instituciones para buscar formas y condiciones de generar recursos y solventarse.

En el caso de Brasil, las alteraciones de la relación entre gobierno nacional y locales, así como la nueva organicidad del sistema educativo, están normatizadas en el FUNDEF, que se constituye en el principal mecanismo de regulación de la municipalización. Este Fondo fue implementado para distribuir los recursos financieros en todo el país, teniendo como objetivo la universalización de la Educación Fundamental.

La política de expansión de la enseñanza, aun siendo común entre los diferentes países de la región, adquiere connotaciones diferentes según el grado de desarrollo de 
la educación formal en cada uno de ellos. En Brasil fue necesario, primero, concentrarse en la universalización de la Educación Fundamental, debido a la escasa cobertura históricamente configurada. En México y en Argentina las políticas pudieron focalizar, desde el inicio, la expansión del nivel medio. En Chile, a su vez, llegaron a proponer, además de esto, la obligatoriedad de la Educación Infantil.

Pasados algunos años de la implantación del FUNDEF, el conjunto de las investigaciones acusa la consolidación de una nueva organicidad del sistema educativo, que contiene una significativa diversidad de situaciones en el país (Viriato 2001; Martins 2004; Bassi 2001; Barreto 1995; Fonseca 1995; Gomes 1995; Oliveira, Duarte 1997).

Las disparidades regionales ya venían acentuándose, resultante de las iniciativas estatales y municipales, y se refuerzan aún más con la lógica de redistribución de responsabilidades entre los diferentes órganos de gobierno y la institución escolar, resultante de la reforma.

Se verificó que el desempeño, el acceso a las informaciones, la capacidad de atender a las demandas educativas, los recursos disponibles y la institucionalización de los órganos de control y gestión son diferentes y desigual en las redes de educación formal existentes en el país (Davis 1999). Esa desigualdad fue reforzada por la disminución de inversiones federales, producto, entre otras cosas, de la transferencia de responsabilidades financieras de las instancias federales que poseían condiciones muy dispares de inversiones y del no cumplimiento, por parte del gobierno federal, del valor del costoalumno estipulado por ley. En contrapartida, la implementación del FUNDEF resultó, en los estados y municipios con menos recursos, en un aumento de los sueldos de los profesores, mientras que los municipios que ya venían invirtiendo en una política salarial de valorización de la categoría fueron perjudicados por el congelamiento y la consecuente reducción de los sueldos docentes (Fracalanza 1999).

La concentración de un porcentaje importante de los recursos financieros en la expansión y mejora de la Educación Fundamental estipulada en el FUNDEF originó, según las investigaciones, resultados antagónicos en la cobertura del sistema educativo. Se obtuvo la casi universalización de la Educación Fundamental en todo el país, con el crecimiento de las redes municipales de ese nivel de enseñanza. Este crecimiento se dio principalmente por la transferencia de las matrículas estatales (Castro 1998), en detrimento de la atención a la Educación Infantil y de Jóvenes y Adultos, que era responsabilidad de los municipios (Gonçalves 1998).

Paralelamente, esa dinámica se sobrepone a las políticas de gestión local que, en consecuencia de la historicidad de sus proyectos políticos locales, continuaron manteniendo una autonomía relativa en fase a los cambios de la regulación de la organicidad del sistema (BOF 1999).

Las investigaciones que centran el análisis de los proyectos políticos locales en sus modalidades de gestión identifican dos concepciones dominantes en el país: la "democrática" y la "gerencialista" (Fonseca 2003; Jacobi 2000; Torres, Garske 2000).

En los municipios que llevaron a cabo un proyecto de gestión democrática, los investigadores identifican que éste tiene como eje principal la autonomía de la escuela, que sería viabilizada con la transferencia de poder de las instancias centrales para las instituciones escolares y con la inclusión de la comunidad educativa en los espacios de decisión. Tales intenciones se expresan en la constitución de mecanismos y prácticas de decisión colectiva que, según verificaron los investigadores, no siempre se hicieron 
efectivos, dada la discontinuidad de estas prácticas. Esto sucede debido a la dificultad de las autoridades locales de convivir con los conflictos y crear una cultura participativa que solvente prácticas colegiadas de gestión, integrando los diferentes segmentos a la dinámica escolar de forma más permanente (Castro 2000; Cardoso 1995; Mattos 1998; Oliveira 2000; Pithan 1995; Souza 2001; Neves 1999). Observan también que el discurso de la descentralización se limite a la desconcentración de las funcionales y a la ausencia de condiciones y orientaciones que hagan efectivas la democratización o que provoquen, realmente, una alteración substantiva en las escuelas en relación a su desempeño (Mendonça 2001; Pavan 1998; Schmidt 2000; Viriato 2001).

Por otro lado, las investigaciones que analizan críticamente la lógica gerencialista vigente en las dinámicas escolares comprenden que las escuelas y las familias son incentivadas a asumir nuevas responsabilidades, sobre el argumento de la autonomía escolar, pero sin los correspondientes insumos materiales, financieros, institucionales y de formación (Fiorini, Filho 1996). Esta suposición está sustentada, según los investigadores, en un discurso participativo que oculta procesos de privatización del servicio público, proceso identificado en los análisis de la dinámica escolar que se establece cuando las escuelas realizan convenios con instituciones privadas para la colaboración en su gestión. En los estudios se observa que la motivación de las empresas es mejorar su imagen social y que los recursos destinados son esencialmente asistenciales (Melo 2001; Souza 2000; Vaini 1999). Los investigadores concluyen, además, que las escuelas obtienen beneficios, principalmente financieros (a pesar de ser momentáneos) y que esos convenios generalmente son frágiles, dada su informalidad.

Los cambios institucionales ocurridos en la gestión escolar produjeron, entre otras cosas, la burocratización de la participación y el aumento de las actividades administrativas (Fernández 1997; Paro 1996), a semejanza de lo que ocurrió en los otros países de la región. Pero en Brasil estos cambios institucionales se entrelazaron, en algunos estados y municipios, con prácticas (vigentes desde la década del 80) que ampliaban la participación de la comunidad escolar. Entre estas, se destaca la designación del cargo de director por el voto directo de la comunidad escolar.

Notaron los investigadores que, en los estados en que prevalecía el clientelismo y el patrimonialismo, la implantación de la elección para director fue reconocida como un avance en el esfuerzo de superación de este histórico comportamiento, a pesar de que en algunos casos estas mismas conductas hayan continuado reproduciéndose, por medio de comportamientos populistas y corporativistas (Paro 1996). También se evidenció que el aumento de la legitimidad de los directores electos y el mayor incentivo a la participación de la comunidad no resultaron en la mejora de la calidad (Cruz, Maia, Vieira 1999; Silva 2001; Teixeira 1998; Castro 1998; Cabral Neto, Almeida 2000) ni en innovaciones en la gestión escolar, conforme a las expectativas que justificaron la implantación de este instrumento.

La descentralización de la administración pública de la educación en Chile se dio, diferentemente a los otros países, en el interior de la lógica unitaria del poder político vigente en el país. Se caracterizó por la creación de instancias administrativas municipales y por la normatización de la participación del sector privado en la gestión pública, permitiéndoles, inclusive, que asumieran la gestión de las instituciones escolares públicas. La descentralización en Chile no ocurrió apenas por la influencia de los organismos internacionales que aportaron recursos, sino también por otros factores específicos de 
la realidad chilena, entre los cuales se destaca la voluntad de la población en volver a participar, lo que había sido interrumpido por la dictadura y el endeudamiento del Estado, a lo que se acrecentaba una burocracia costosa, por eso el énfasis en compartir con la sociedad civil la inversión en educación (Moreira 1999).

La política de municipalización contempló la transferencia de responsabilidades y competencias del gobierno central para los municipios y la creación de instancias administrativas colegiadas de gestión municipal. Previa, además, la elaboración de un plan educativo por el conjunto de la red local (Espinoza 1997; Navarro 2000). Sin embargo, las investigaciones mostraron que la municipalización se redujo a la transferencia de la administración de servicios educativos para los municipios, excluyéndose las funciones técnico-pedagógicas, que permanecieron en las manos del Ministerio y de las respectivas secretarías regionales y dirección provincial (Navarro 2000; Paredes 1999).

Observan, además, que la consolidación de una propuesta colectiva para el municipio se mostró inviable, porque muchas veces éstos no daban prioridad a la educación en el rol de políticas públicas o poseían la competencia técnica necesaria, y también porque su consolidación presuponía una rápida movilización de las escuelas para la tomada de decisiones y proyecciones futuras (Mineduc 2001).

La normatización de la participación del sector privado en la gestión pública se concretizó principalmente en la reconfiguración de la lógica institucional de la escuela pública, a través de la reglamentación del sistema de subvenciones. Se configura, así, un sistema compuesto por escuelas municipales, subvencionadas, privadas y privadas-subvencionadas (Mizala, Romanega 1998; González 1999; Miranda, De Simone 2002).

Además de los cambios en la lógica de la gestión escolar, la incorporación de la iniciativa privada en la gestión pública de la educación propiciaría una correlación de fuerzas favorable a sus intereses en la definición de un proyecto nacional. Esta nueva lógica no cambia apenas las relaciones en el interior de las unidades escolares como altera el carácter de la participación de la comunidad (Servat 1996; Reca 2002).

Según los investigadores, la reconfiguración de la relación del Estado con la sociedad transformó la tradicional cultura de participación colectiva (Gubbins 2001), ya fragilizada por el gobierno militar, en una cultura de participación privada, que defiende sus derechos como consumidor (Carnoy 1998; Reca 2002).

Esta nueva organicidad que acabamos de mencionar fue asumida por el gobierno nacional como la modernización de la educación en pro de la equidad y de la calidad (Bellei 2001). Sin embargo, como advierten los investigadores, este propósito no se confirmó, pues las escuelas desarrollaron comportamientos discriminatorios para garantizar la obtención de la subvención condicionada al desempeño de los alumnos (Sanhueza 1999, Carnoy 1998). De esta forma, en las subvencionadas se generó un mecanismo de privilegios a estudiantes con mejores condiciones culturales y económicas. En consecuencia, las instituciones con menos recursos, principalmente las municipales, pasaron a atender a los estudiantes con dificultades de aprendizaje y, en general, de familias con menores condiciones socioeconómicas y con menos posibilidades de invertir en la institución. Se refuerza, así, la discriminación y la desigualdad en el interior del sistema educativo (Serrano, Fernández 2001; Figueroa 1997).

Esta reproducción de la desigualdad es observada por los investigadores también cuando analizan la implantación de la Jornada Escolar Completa (JEC). Resaltan que 
para ser válida exige una nueva organización interna de los establecimientos y una infraestructura mínima para soportar toda la demanda (Herrera 1996).

El proceso requiere aún un nuevo tipo de trabajo docente y, en consecuencia, mayor disponibilidad de tiempo, ya que el profesor tendrá que adaptar sus planes de trabajo a cada realidad. Consecuentemente con este análisis, la falta de recursos financieros (para cubrir los gastos con infraestructura, alimentación, inversión en cursos de capacitación de los docentes) constituye uno de los mayores problemas en la consolidación de la JEC, cuyo suceso depende del aumento de la subvención estatal a las escuelas.

Todas estas constataciones no invalidan el consenso en torno de la descentralización del sistema educativo. Con todo en el interior de ésta, los investigadores observaron una tensión social entre los defensores de mayor y los de menor reglamentación de la autonomía de las escuelas subvencionadas en relación al gobierno central.

Es común encontrar en las soluciones alternativas propuestas por algunos autores para los problemas detectados el cuestionamiento de las normatizaciones asumidas en los últimos años. En estos casos, se apunta que las deficiencias del sistema derivan de mecanismos de control aún centralizados en las manos del gobierno nacional (Paredes 1999). A este respecto, también aparece el siguiente razonamiento: cuando este aumento de responsabilidades viene acompañado de recursos públicos o privados, parece estimular acciones vinculadas a la liberalización de las normas, para no ser sometidos a ningún control. En contrapartida, cuando el aumento de responsabilidades no viene acompañado del correspondiente aumento de recursos se solicita la presencia del Estado (Bravo, s/d, Cardona 2000).

El hecho de que el sector privado asuma responsabilidades y atribuciones en la gestión de la educación pública demuestra diferentes grados de consolidación, según la tradición de participación de este sector. El reordenamiento del sistema de subvenciones en Chile que, como vimos, institucionaliza la participación de la iniciativa privada en la gestión de la educación pública, da continuidad a una política de apertura para el mercado en la gestión del espacio público.

\section{CONCLUSION}

A lo largo de este texto, cuando analizamos los aspectos comunes y las especificidades en los cambios ocurridos en la organicidad del sistema educativo en Argentina, Brasil, Chile y México, nos encontramos con una diversidad en el interior de la homogeneización aludida en el debate regional. Las especificidades de las realidades sociales se presentan en las investigaciones, principalmente a través de sus categorías de análisis que, al mismo tiempo, van a revelarse en el carácter del conocimiento resultante.

Además de la relación entre la producción intelectual y la base social de donde emergen y con la cual interactúan, encontramos también la vinculación históricamente configurada de las tendencias nacionales e internacionales produciendo impactos diferentes de la reforma en los países.

El proceso de inducción externa de la reforma, desencadenada en el inicio de la década del 90, se configuró de forma particular en cada país según los embates políticos nacionales por la construcción de una nueva hegemonía, que se expresan en una nueva normatividad de la política educativa en la década siguiente. Esos choques de posiciones 
tornaron públicas las expectativas y los diferentes intereses presentes en los escenarios nacionales que movilizaron las sociedades hasta inicios de la década del 90 cuando, entonces, consolidando el predominio de algunos sectores, se observó la centralización de las decisiones político-educativas en el ámbito gubernamental, restringiendo sus negociaciones a algunos sectores nacionales e internacionales.

A partir de allí, conforme a las reflexiones presentadas en este trabajo, se instaló en América Latina, a lo largo de los años 90, un nuevo modelo de organización y gestión de sistema y de sus instituciones. Esto nos presenta, en el umbral del siglo XXI, un escenario educativo que, sobre el fetiche de la modernidad y de la democratización, respondió a la necesidad de adecuación de la gestión de la educación pública a los cambios de la lógica de regulación instaurada por la nueva etapa del capitalismo. Este escenario, revelado por las investigaciones en cada uno de los países, nos permite sostener la hipótesis de que el centro de la reforma no fue la institución escolar, tal como es proclamado, sino la lógica y las instancias de regulación social. Por el contrario, el foco de la transformación fue el gobierno central, con las adecuaciones que se hicieron necesarias en las demás instancias de decisión gubernamental, inclusive en la institución escolar.

Se observa el extrañamiento ${ }^{5}$ de la dinámica institucional concretizada al configurarse contradictoria a las finalidades de la reforma educativa, legitimadas en la lógica capitalista contemporánea, sobre el fetiche de la modernidad y de la democratización. De esta manera se acentúan problemas que supuestamente se pretendían eliminar como, por ejemplo, frente a la intencionalidad explícita de socialización equitativa del conocimiento se observa el aumento de la desigualdad.

Las directrices educativas en cada país demostraron diferentes grados de concretización, conforme las particularidades de los poderes ejecutivos y sus alianzas, la ruptura o la continuidad frente a proyectos políticos anteriores (que le confirieron diferentes grados de legitimidad) y la movilización y resistencia de diversos segmentos de la sociedad.

Lo que se observa es que la historicidad que caracteriza la realidad educativa en estos países sufrieron en México lo que podríamos denominar una "ruptura conservadora", por su carácter regresivo; en Chile, una "continuidad conservadora", dado el mantenimiento y ampliación de las propuestas de cuño neoliberal; en Brasil, una renovación conservadora, por su activismo innovador, y en Argentina una ruptura interrumpida, del estancamiento de las renovaciones pretendidas.

El "nuevo conservadorismo" en la realidad educativa expresa la finalidad común apuntada anteriormente, en cuanto la historicidad vigente en cada país se revela en la especificidad de sus metas y de sus estrategias, dentro de las cuales destacamos la expansión de la enseñanza, la desregulación del trabajo docente y la configuración de las responsabilidades en el ámbito educacional.

5 El extrañamiento, concepto concebido por Marx, es inherente a la alienación y se manifiesta en el interior del proceso de producción capitalista en el cual el objeto producido enfrenta al productor como ser extraño, como un poder independiente de él. 


\section{BIBLIOGRAFIA}

Aboites, H. La propuesta de educación básica del gobierno de Vicente Fox: un análisis desde el Programa de Escuelas de Calidad. Xochimilco, Ciudad de México: Universidad Autónoma Metropolitana, Departamento de Educación y Comunicación, s/d.

Arroyo, M. (2004). ¿Hay en la escuela algo que tenga que ver con un proyecto común? Reflexiones sobre las relaciones entre las políticas, la solidaridad y la escuela. En: Tiramonti, G. La trama de la desigualdad educativa: mutaciones recientes en la escuela media (p. 144-146). Buenos Aires: Manantial.

Baeza Narciso, P. (1997). Pariente pobre de las estrategias educativas: todo para el pueblo, pero sin el pueblo. Santiago: Disert. (maest.) Facultad de Educación, Universidad de Chile.

Barreto, E. S. de S. (1995). Descentralizar e redistribuir nos sistemas de ensino. San Pablo: $\mathrm{Ca}$ dernos de Pesquisa $\mathrm{N}^{\circ}$ 95: 73-78.

Barreyro, G. B. (2001). Políticas educativas en la Argentina a fines del siglo XX: un estudio del Plan Social Educativo. Buenos Aires. Disert. (maest.) Faculdad Latinoamericana de Ciencias Sociales.

Bassi, M. (1996). Política educacional e descentralização: uma crítica a partir da análise da descentralização financeira dos recursos públicos enviados às escolas no Estado de Minas Gerais. San Pablo. Disert. (maest.) Faculdad de Educación da Pontifícia Universidad Catolica de San Pablo.

Bassi, M. (2001). Gestão e financiamento da educação básica: repercussões do Fundef em sete municípios paulistas. San Pablo. Tese (dout.) Pontifícia Universidad Catolica de San Pablo.

Belleï, C. (2001). ¿Ha tenido impacto la Reforma Educativa chilena? Revista Educación, No 291 : $29-40$.

Bezerra, A. A. (1997). Gestão democrática da construção de uma proposta curricular no ensino público: a experiência de Aracaju. San Pablo. Tese (dout.) Faculdad de Educación, Universidad de San Pablo.

Birgin, A. (2000). Novas regulações do trabalho docente: o caso da Argentina. San Pablo: Cadernos de Pesquisa $\mathrm{N}^{\circ}$ 111: 95-113.

Bof, A. M. (1999). Mudando a gestão da escola para melhorar a qualidade: o caso de Rondonópolis. Rio de Janeiro: Ensaio v. 7, $\mathrm{N}^{\circ} 25$.

Bonilla, R. (2002). Programa de Transformación de la Gestión Escolar. Ciudad de México: Subsecretaría de Educación Básica y Normal, Dirección General de Investigación Educativa. (Documento de Trabajo).

Bracho, T. (1997). Educación básica en México (1990-1996). Ciudad de México: Cide, División de Administración Pública $\mathrm{N}^{\mathrm{o}} 71$.

Bravo, D.; A. Marinovic. La educación en Chile: una mirada desde la economía. Santiago: Universidad de Chile, Facultad de Ciencias Económicas y Administrativas, s/d.

Cabral Neto, A.; M. D. Almeida (2000). Educação e gestão descentralizada: conselho diretor, caixa escolar, projeto político-pedagógico. Brasília: Em Aberto v. 17, № 72 .

Calvo Pontón, B. (1997). La modernización educativa: una perspectiva regional desde la frontera norte de México. Ciudad do México. Tesis (doct.) Universidad Iberoamericana.

Calvo Pontón, B. et al. (2002). La supervisión escolar de la educación primaria en México: prácticas, desafíos y reformas. París: lipe/UNESCO.

Camacho, S. S. (2000). Resistencia sindical a la descentralización educativa: el caso Aguascalientes, 1978-1993. Aguascalientes: Caleidoscopio $\mathrm{N}^{\circ}$ 7: 7-48.

Camacho, S. S. Modernización educativa en México, 1982-1998: el caso de Aguascalientes. Aguascalientes: Universidad Autónoma de Aguascalientes, Instituto de Educación, s/d.

Cardona Y. L. D. (2000). Políticas educacionales para grupos vulnerables: lecciones de la experiencia chilena. Santiago: Tesis (doct.) Facultad de Ciencias Físicas y Matemáticas, Universidad de Chile.

Cardoso, J. (1995). Gestão compartilhada da educação: a experiência catarinense. Brasília: Revista Brasileira de Estudos Pedagógicos v. 76. 
Carmona, A. L. (1996). Proceso de desconcentración de la educación básica en el Distrito Federal. Ciudad de México: Tesis (doct.) Facultad de Ciencias Políticas y Sociales, Universidad Nacional Autónoma de México.

Carnoy, M. (1998). National Voucher Plans in Chile and Sweeden: did privatization reforms make for better education? Chicago: Comparative Education Review v. 42, $\mathrm{N}^{\mathrm{o}} 3$.

Castro, J. A. (1998). Federalismo e gasto público. Brasília: Revista Brasileira de Estudos Pedagógicos v. 79: $\mathrm{N}^{\circ}$ 192: 92-100.

Castro, J. A. (1998a). Gastos públicos com a educação básica. Brasília: Revista Brasileira de Estudos Pedagógicos v. 79, $\mathrm{N}^{\circ}$ 193: 113-134.

Castro, M. L. S. (2000). A gestão da escola básica: conhecimento e reflexão sobre a prática cotidiana da diretora de escola municipal. Brasília: Em Aberto v. 17, No 72: 71-87.

Costa, V. L. C. et al. (1997). Gestão educacional e descentralização: novos padrões. São Paulo: Cortez.

Cruz, S. H. V.; M. H. Maia, S. L. Vieira (1999). Eleição de diretores: uma mudança na cultura escolar. Porto Alegre: Revista Brasileira de Política e Administração da Educação v. 15, № 2.

Davies, N. (1999). O Fundef e o orçamento da educação: desvendando a caixa preta. Campinas: Autores Associados.

Díaz, A. B. (2002). Los procesos de frustración en la tarea docente. Santiago: Revista Docencia, Colegio de Profesores de Chile v. 17, $\mathrm{N}^{\circ}$ 17: 64-76.

Donoso Díaz, S. (2004). Balance y perspectivas de quince años de política y Reforma Educativa en Chile. Santiago (mimeo).

Duschatzky, S. (coord.) (1999). Aproximaciones al proceso de reforma en el tercer ciclo de la Provincia de la Pampa. Buenos Aires: Faculdad Latinoamericana de Ciencias Sociales.

Duschatzky, S., P. Redondo (2000). El Plan Social Educativo o los indicios de ruptura de las políticas públicas. In: Dustchatzky, S. (comp.). Tutelados y asistidos: programas sociales, políticas públicas y subjetividad. Buenos Aires: Paidós.

Dussel, I., G. Tiramonti, A. Birgin (1998). Nuevas tecnologías de intervención en las escuelas. Programas y proyectos. Buenos Aires: Propuesta Educativa, Flacso No 18.

Escoffier, M. T. (1995). Esos cuatro millones de pobres: la educación significa inversión social. Santiago: Revista de Educación, Mineduc v. 7, № 224.

Espinoza, J. J. (1997). Desarrollo y gestión educativa. Santiago: Revista Economía y Administración, Cpeip No 132: 65-75.

Espinoza, V. A. V. (coord.) (1999). Modernización Educativa y cambio institucional en el Norte de México. Baja California: El Colegio de la Frontera Norte.

Esquivel, G. H. (1999). Educación y desarrollo regional: una evaluación de aportaciones para la educación básica y normal. Ciudad de México: Momento Económico № 104: 13-20.

Ezpeleta, J. (1997). Reforma educativa y zonas de turbulencia. Ciudad de México: DIE, Cinvestav.

Ezpeleta, J. (2003). Cambio pedagógico sin cambio institucional en la escuela primaria: a propósito de la innovación (memória eletrônica). Ciudad de México: Comie (Ponencia a $7^{\circ}$ Congreso Nacional de Investigación Educativa).

Ezpeleta, J., E. Weiss (2000). Cambiar la escuela rural. Ciudad de México: DIE, Cinvestav.

Fernandes, M. D. E. (1997). Política educacional: a gestão democrática na rede estadual de ensino em Mato Grosso do Sul (1991 a 1994). São Paulo: Fundação Carlos Chagas.

Figueroa, A. R. (1997). Da resignação ao consentimento: privatização da educação média no Chile. São Paulo: Cadernos de Pesquisa $\mathrm{N}^{\circ} 100$.

Fiorini Filho, J. A. (1996). Gestão da escola pública: o diretor em sua ação cotidiana. San Pablo: Dissert. (maestr.) Faculdad de Educación da Pontifícia Universidad Católica de San Pablo.

Fonseca, J. P. (1995). Poder local e municipalização: em busca da utopia; um estudo do município de Jacuí (MG). San Pablo: Tesis (livre-doc.). Faculdad de Educación da Universidad de San Pablo.

Fonseca, M. (2003). A participação escolar no contexto do PDE. In: Fonseca, M. (coord.). Novos modelos de gestão da educação básica: o que mudou na escola? Brasília: UNB. 
Fracalanza, P. S. (1999). A gestão do ensino fundamental pelo governo do Estado de São Paulo: uma análise do financiamento e dos indicadores sociais de educação (1980-1993). Campinas: Educação e Sociedade V. 20, $\mathrm{N}^{\circ}$ 69: 92-118.

García-Huidobro, J. E. (ed.) (1999). La Reforma Educacional chilena. Madrid: Popular.

García-Huidobro, J. E. (mar. 2004). La equidad y la igualdad en la educación chilena: comentários al capítulo 7 de revisión de políticas nacionales de educación. Santiago: OCDE, Universidad Alberto Hurtado.

García-Huidobro, J.E., C. Cox (1999). Visión de conjunto. In: García-Huidobro, J. E. (ed.). La Reforma Educacional chilena. Madrid: Popular.

GOMES, A. A. J. (1995). Municipalização do ensino: da falácia utópica ao realismo competente. Rio de Janeiro: Tecnologia Educacional v. 24, № 122: 7-11.

Gonçalves, R. (1998). O financiamento da educação: um sistema em desequilíbrio. San Pablo: Cadernos de Pesquisa $\mathrm{N}^{\circ}$ 103: 14-15.

González, P. (1999). Financiamiento, incentivos y reforma educacional (pp. 23-26). En: GarcíaHuidobro, J. E. (ed.). La Reforma Educacional chilena. Madrid: Popular.

González, S. N. De S.; A. Arango (1997). Educación en Argentina: ¿centralizando la descentralizatión y reformando la Reforma? São Bernardo do Campo Revista Brasileira de Política e Administração da Educação v. 13, № 1: 71-106.

Gubbins, V. F. (2001). Relación entre escuelas y familias: estado presente y desafíos pendientes. Provincia de Antofagasta: Estudios Sociales $N^{\circ} 108$.

Guimarães, J. (2004). Alguns impactos do Fundef: apontamentos e incertezas após a sua implantação (p.193-210). In: Martins, Â. M.; Oliveira, C. de; Bueno, M. S. S. (org.) Descentralização do Estado e municipalização do ensino: problemas e perspectivas. Rio de Janeiro: DP\&A.

Herrera, R. (1996). Reforma Educacional: jornada escolar completa diurna. Santiago: Colegio de Profesores de Chile.

Illanes, M. C.; M. Lagos (1998). Reforma Educacional: a propósito de una encuesta. Revista de Educación $\mathrm{N}^{\circ} 260$.

Jacobi, P. (2000). Educação, ampliação da cidadania e participação. San Pablo: Educação e Pesquisa v. $26, \mathrm{~N}^{\circ} 2$.

Kessler, G. (2002). La experiencia educativa fragmentada: estudiantes y profesores en las escuelas medias de Buenos Aires. Buenos Aires: lipe, UNESCO.

Kisilevsky, M. Federalismo y educación: un espacio histórico de pugnas distributivas. Buenos Aires, s/d. Disert. (maest.) Faculdad Latinoamericana de Ciencias Sociales.

Krawczyk, N. R.; V. L. Vieira (2005). O estado da arte das pesquisas sobre a reforma educacional na década de 90, na Argentina, Brasil, Chile e México. São Paulo: Fapesp. (Documento Final).

Krichesky, M.; I. Cappellarcci (1999). Gestión curricular y riesgo pedagógico en EGB3: un análisis de casos en Lomas de Zamora y La Matanza. Propuesta Educativa. Flacso No 21: 66-79.

Latapí Sarre, P.; M. Ulloa (2000). El financiamiento de la educación básica en el marco del federalismo. Ciudad de México: Centro de Estudios sobre la Universidad de la Unam. (Sección de Obras de Educación y Pedagogía).

Loera, A. V. (2003). Reporte descriptivo de la línea de base de la evaluación cualitativa del Programa Escuelas de Calidad. Secretaría de Educación Pública, Subsecretaría de Educación Básica y Normal. Ciudad de México: Heurística Educativa.

Loera, A.; F. S. Sandoval (1999). La innovación en el proceso de descentralización en el estado de Chihuahua. En: Pardo, M. del C. (coord.) Federalización e innovación educativa en México. Ciudad de México: El Colegio de México.

Loyo, A.; A. Muñoz (2001). La concertación de las políticas educacionales: el caso de México. Ciudad de México: Instituto de Investigaciones Sociales de la UNAM, Universidad Iberoamericana (Documento de trabajo).

Martínez García, R. (coord.); J. L. Cuéllar Romero (2003). El Programa Escuelas de Calidad en la educación primaria. San Luis de Potosí: Secretaría de Educación del Gobierno del Estado, Coordinación General de Validación. 
Martins, M. A. (2004). O processo de municipalização no Estado de São Paulo: um novo desenho da política educacional (p. 151-177). In: Martins, Â. M.; Oliveira, C.; Bueno, M. S. S. (org.) Descentralização do Estado e municipalização do ensino: problemas e perspectivas. Rio de Janeiro: DP\&A Ed.

Mattos, L. A. F. (1998). Democracia e colegiado de escola: qual democracia? Um estudo da gestão colegiada em Minas Gerais. Rio de Janeiro: Tésis (dout.) Faculdad de Educación, Universidad Federal del Rio de Janeiro.

Melo, M. T. L. (2001). Gestão educacional: os desafios do cotidiano escolar (p.243-254). En: Ferreira, N. S. (org.). Gestão da educação, impasses, perspectivas e compromissos. São Paulo: Cortez.

Mendonça, E. F. (2000). A gestão democrática nos sistemas de ensino brasileiros: a intenção e o gesto (p. 24-28). In: Reunião Anual Da Anped, 23. Caxambu.

Mercado, R. M. (1997). La educación primaria gratuita, una lucha popular cotidiana. Ciudad de México: DIE, Cinvestav (Serie Tesis).

Mineduc (2001). La escuela busca el compromiso de los padres. Santiago. Revista de Educación $\mathrm{N}^{\circ}$ 284. Mineduc.

Miranda Sales, E.; J. De Simone Lavia (2002). Gestión educacional descentralizada: análisis y propuestas de acción a nivel municipal. Concepción, Chile: Universidad San Sebastián.

Mizala, A.; P. Romaguera (2000). Sistemas de incentivos en educación y la experiência del Sned en Chile. Santiago: Centro de Economía Aplicada, UCL. (Documento de trabajo, 82).

Mizala, A.; P. Romaguera (1998). Desempeño escolar y elección de colegios: la experiencia chilena. Santiago: Centro de Economía Aplicada, Departamento de Ingeniería Industrial, Facultad de Ciencias Físicas y Matemáticas, UCL. (Documento de trabajo, 38).

Mizala, A.; P. Romaguera, D. Fareen (1998). Eficiencia técnica de los establecimientos educacionales en Chile. Santiago: Centro de Economía Aplicada, Departamento de Ingeniería Industrial, Facultad de Ciencias Físicas y Matemáticas, Universidad de Chile. (Serie Economía, 38).

Monzón T., L. A. (2000). La Reforma Educativa chilena bajo la percepción del profesor de aula. Santiago: Disert. (maestr.). Facultad de Filosofía y Educación, Universidad Metropolitana de Ciencias de la Educación.

Moreira Tapia, F. (1999). Descentralización y calidad de los aprendizajes en escuelas municipales chilenas. Santiago: Disert. (maestr.) Facultad de Educación, Pontificia Universidad Católica de Chile.

Navarro, L. N. (2000). La autoevaluación como herramienta para el control y mejoramiento de la gestión de los establecimentos municipales de educación. Santiago: Disert. (maest.) Facultad de Educación, Universidad Católica de Chile.

Neves, L. M. V. (1999). Um caminhar para um mesmo lugar (p.134-135). In: Lesbaupin, I. (org.). $O$ desmonte da nação: balanço do governo FHC. Petrópolis, Vozes.

Núñez, I. (1999). Antecedentes históricos de la actual renovación pedagógica en la educación chilena. Santiago (mimeo).

Núñez, I. (1994). Modernizaciones en la educación chilena en siglo XX. Santiago (Apresentada em sessão de la Sociedad Chilena de Historia da Educación).

Núñez, I. (1999a). Peculiaridad de la Reforma chilena. Santiago (mimeo).

Núñez, I. (nov. 1999b). La política educacional del régimen militar. Santiago (mimeo).

Oliveira, C. (2004). Algumas observações sobre o financiamento do ensino (p.129-143). In: Martins, Â. M.; Oliveira, C.; Bueno M. S. S. (org.). Descentralização do Estado e municipalização do ensino: problemas e perspectivas. Rio de Janeiro, DP\&A.

Oliveira, D. A.; M. R. T. Duarte (1999). Política e trabalho na escola: administração dos sistemas públicos de educação básica. Belo Horizonte: Autêntica.

Oliveira, D. A.; M. R. T. Duarte (1997). Políticas e administração da educação: um estudo de algumas reformas recentes implementadas no Estado de Minas Gerais. Campinas: Educação e Sociedade $\mathrm{N}^{\circ}$ 58: 123-141.

Oliveira, M. A. M. (1997). Qualidade, parceria e redenção: o projeto-piloto de implantação da qualidade total em uma escola estadual de Belo Horizonte. Campinas: Tesis (dout.) Faculdad de Educación, Universidad Estadual de Campinas. 
Oliveira, N. C. M. (2000). A política educacional no cotidiano escolar: um estudo meso-analítico da organização escolar em Belém-PA. San Pablo: Tesis (dout.) Pontifícia Universidad Católica de San Pablo.

Ornelas, C. (1999). La descentralización de la educación en México: el federalismo difícil. En: Cabrero, E. (comp.). Las políticas descentralizadoras en México (1983-1993): logros y desencantos. Ciudad de México: Miguel Ângel Porrúa.

Ornelas, C. (coord.) (2002). Valores, calidad y educación: memoria del primer encuentro internacional de educación. México: Santillana.

Oyola, C. (dir.) (1998). Innovaciones educativas: entre las políticas públicas y la práctica educativa: un análisis de la Reforma Educativa del nivel medio en Río Negro (1986-1996). Buenos Aires: Miño y Dávila.

Pardo, M. (coord.) (1999). Federalización e innovación educativa en México. Ciudad de México: El Colegio de México.

Paredes Villegas, C. (1999). La descentralización de la administración pública: el proceso de municipalización democrática del sistema educacional chileno. Santiago: Disert. (maest.) Universidad Académica de Humanismo Cristiano.

Paro, V. (1996). Eleição de diretores de escolas públicas: avanços e limites da prática. Brasília: Revista Brasileira de Estudos Pedagógicos v. 77, № 186.

Pavan, R. (1998). A municipalização do ensino fundamental: o caso de Santos e Jundiaí. San Pablo: Disert. (maest.) Programa História e Filosofia da Educação, Pontifícia Universidad Católica de San Pablo.

Pereira, S. Petruzzi (2000). Disposición de los docentes de la comuna de La Granja frente a los ámbitos de la Reforma chilena. Santiago: Disert. (maest.) Facultad de Filosofía y Educación, Universidad Metropolitana de Ciencias de la Educación.

Pithan, N. de A. (1995). O cotidiano escolar e o Conselho de Classe: uma abordagem simbólica e ritual. Rio de Janeiro: Disert. (maest.) Faculdad de Educación, Universidad Federal del Rio de Janeiro.

Poliak, N. (2004). Reconfiguraciones recientes en la educación media: escuelas y profesores en una geografía fragmentada. En: Tiramonti, Guillermina. La trama de la desigualdad educativa: mutaciones recientes en la escuela media. Buenos Aires: Manantial.

Prado, M. N., M. Gajardo (1995). Educación rural: emergiendo desde lo propio. Qué poco sabíamos de la escuela rural. Santiago: Revista de Educación $\mathrm{N}^{\circ} 227$.

Puiggrós, A. (1990). Sujetos, disciplina y currículo en los orígenes del sistema educativo argentino. Buenos Aires: Galerna.

Quiroz, R. (2000). Las condiciones de posibilidad de aprendizaje de los adolescentes en la educación secundaria. Ciudad de México: Tesis (dout.) DIE-Cinvestav.

Reca, I. (2002). La participación organizada de los padres en la educación. Santiago: Revista Cuadernos Sociológicos $\mathrm{N}^{\mathrm{o}} 1$.

Riquelme, G. (2004). La educación secundaria antes y después de la Reforma: efectos distributivos del gasto público. Buenos Aires: Miño y Dávila.

Rivas, A. (2004). Gobernar la educación: estudio comparado sobre el poder y la educación en las provincias argentinas. Buenos Aires: Granica.

Rockwell, E. (1997). Escuelas comunitarias: una primera alternativa para el medio rural. Santa Fé de Bogotá: Revista Colombiana de Educación $\mathrm{N}^{\mathrm{o}} 34$.

Romagnoli, M. C.; E. R. Romagnoli et al. (2002). La implementación del octavo año de la EGB en la Provincia de Mendoza. Mendoza.

San Miguel, J. (1999). Programa de Educación Básica Rural. En: García-Huidoro, J. E. (ed.) La Reforma Educacional chilena. Madrid: Ed. Popular.

Sandoval, E. F. (2002). La trama de la escuela secundaria, instituciones, relaciones y saberes. Ciudad de México: Plaza y Valdés.

Sanhueza, C. (1999). Rendimiento educacional, desigualdad y brecha de desempeño privado y público: Chile 1982-1997. Santiago: Facultad de Ciencias Económicas y Administrativas, Universidad de Chile. Tesis (maestría). 
Schimitd, W. (2000). A municipalização do ensino fundamental em dois pequenos municípios rurais de Santa Catarina: Anitápolis e Santa Rosa de Lima (1987-95). San Pablo: Tesis (doct.) Pontifícia Universidad Católica de San Pablo.

Schmelkes, S. (1995). Problemas y retos de la educación básica en México. En: Sierra, M. T. (coord.) Cambio estructural y modernización educativa. Ciudad de México: UPN, UAM, Comecso.

Serrano, C., I. Fernández (2001). Antecedentes teóricos e históricos sobre el processo de descentralización educacional en Chile. Santiago: Revista Estudios Sociales, Ed. Corporación de Promoción Universitária, No 107.

Servat P., B. (1996). Participación y eficacia en la escuela: estudios empíricos. Santiago: Disert. (maest.) Facultad de Educación, Universidad de Chile.

Silva, A. A. (2001). Contornos da gestão do ensino fundamental em Feira de Santana (1985-1996). Rio de Janeiro.

Sousa, S. Z. L. (2000). Parceria escola-empresa no Estado de São Paulo: mapeamento e caracterização. Campinas. Educação e Sociedade v. 21, No 70.

Souza, R. De C. V. M. (2001). A gestão do sistema público de ensino do Distrito Federal no período de 1995 a 1998: uma gestão praxística e multirreferenciada? Rio de Janeiro: Anped.

Street, S. (2001). Modernización administrativa o democratización sindical: nuevas jerarquías o nuevos sujetos como vías de Reforma Educativa (p.185-169). En: Educación y Reforma Constitucional. Ciudad de México: Grupo Parlamentario del PRD (mimeo).

Tedesco, J. C. (1982). Educación y sociedad en Argentina (1800-1900). Buenos Aires: Centro Editor de América Latina. (Serie complementar: Educación y Cultura).

Tedesco, J. C.; E. Tenti Fanfani (2001). La Reforma Educativa en la Argentina: semejanzas y particularidades. Buenos Aires: IIPE.

Teixeira, L. H. G. (1998). Cultura organizacional e projeto de mudança em escolas públicas: um estudo de escolas da rede estadual de Minas Gerais. Campinas: Tesis (doct.) Faculdad de Educación, Universidad Estadual de Campinas.

Tiramonti, G. (2001). Modernización educativa de los 90: ¿el fin de la ilusión emancipadora? Buenos Aires: Flacso, Temas.

Tiramonti, G. (2004). La fragmentación educativa y los cambios en los factores de estratificación. En: Tiramonti, G. La trama de la desigualdad educativa: mutaciones recientes en la escuela media. Buenos Aires: Manantial.

Torres, Á.; L. M. Garske (2000). Diretores de escola: o desacerto com a democracia. Brasília: Em Aberto v. 17, $\mathrm{N}^{\circ} 72$.

Vaini, S. S. (1999). Parceria, os perigos da educação (com)partilhada: a influência da iniciativa privada no currículo de escola pública municipal de São Paulo. San Pablo: Disert. (maestr.) Pontifícia Universidad Católica de San Pablo.

Vargas, P.; H. Vargas (1997). El financiamiento compartido y la calidad de la educación. Santiago: Revista Mensaje. Impresión Salesiana v. 46, N 445: 61-63.

Vior, S. (dir.) (1999). Estado y educación en las provincias. Buenos Aires: Miño y Dávila.

Viriato, E. O. (2001). Política educacional do Estado de São Paulo (95 a 98): desconstrução da escola pública. San Pablo: Tesis (doct.) Pontifícia Universidad Católica de San Pablo.

Weinberg, G. (1987). Modelos educacionais no desenvolvimento histórico da América Latina (p.17-45). In: SAVIANI, D. et al. Desenvolvimento e educação na América Latina. São Paulo: Cortez. (Coleção Polêmicas de nosso Tempo). 